\title{
Enhancements in 3D dosimetry measurement using polymer gel and MRI
}

\begin{abstract}
The effects of varying the concentrations of cross-linker $\mathrm{N}, \mathrm{N}$-methyelene-bis-acrylamide (BIS) from $2 \%$ to $4 \%$, and 2-hydroxyethylacrylate(HEA) monomer from $2 \%$ to $4 \%$ at $5 \%$ gelatin on the dose response of BIS-HEA-gelatin (BHEAG) aqueous polymer gel dosimeters have been studied using magnetic resonance imaging (MRI) for relaxation rate (R2) of water proton. The dosimeters were irradiated with 60Co teletherapy -ray source at a constant dose rate, receiving doses up to $30 \mathrm{~Gy}$. The radiation polymerization occurs and increases with increasing initial dose. R2 is found to decrease mono-exponentially with depth inside the polymer gel and depend strongly upon the initial concentrations of co-monomers (HEA and BIS). Dose-depth map for BHEAG gel was determined for different concentrations of comonomer (HEA and BIS). The percentage dose depth was also evaluated which leads to a good agreement with the ionization chamber measurements.
\end{abstract}

Keyword: Polymer gel, Radiation, Relaxation rate, Depth-dose, Cross-linker 